\title{
Study of the Wolf-Rayet population in NGC 604 using bi-dimensional spectroscopy
}

\author{
Jesús Maíz-Apellániz ${ }^{1,2}$, José Miguel Mas-Hesse ${ }^{1}$, and Enrique Pérez ${ }^{3}$ \\ ${ }^{1}$ LAEFF-INTA, PO Box 50727, 28080 Madrid, Spain \\ ${ }^{2} U A X, 28691$ Villanueva de la Cañada, Madrid, Spain \\ ${ }^{3} I A A-C S I C$, PO Box 3004, 18080 Granada, Spain
}

\begin{abstract}
We study the properties of the young population of NGC 604 and find evidences for 1-2 Myr differences between different regions. Four of the eleven WR stars in NGC 604 are found to have wide $\mathrm{H} \alpha$ emission lines. None of the WR stars is located within knots of high density, high excitation gas, as opposed to some of the O-type stars.
\end{abstract}

\section{Observations}

We have mapped spectrophotometrically the giant H II region NGC 604 in M 33 by placing the long-slit of the IsIs spectrograph of the wht at ten different positions. The spectra were obtained at a P.A. of $90^{\circ}$ with an effective width for each one of $1^{\prime \prime}$ and a spacing of $2^{\prime \prime}$ to $3^{\prime \prime}$ between the center of each two consecutive positions. Two spectra were taken simultaneously at each position, one in the range between 6390 and $6840 \AA$ (red arm) and the other one in the range between 4655 and $5055 \AA$ (blue arm), both of them with a dispersion of $\sim 0.4 \AA /$ pixel. Synthetic bi-dimensional maps of five line-ratios $(\mathrm{H} \alpha / \mathrm{H} \beta$, [S II $] \lambda 6717 /[\mathrm{S} \mathrm{II}] \lambda 6731$, ([S II] $\lambda 6717+[\mathrm{S} \mathrm{II}] \lambda 6731) / \mathrm{H} \alpha,[\mathrm{O} \mathrm{III}] \lambda 5007 / \mathrm{H} \beta$ and $[\mathrm{N} \mathrm{II}] \lambda 6584 / \mathrm{H} \alpha)$ were produced. We searched for the spectral signature of WR stars in the form of the WR bump at $\lambda 4686 \AA$ and wide $\mathrm{H} \alpha, \mathrm{H} \beta$ and $\mathrm{He}$ I $\lambda 6678$ emission lines. The above observations were completed with HST-WFPC 2 archival images obtained under five different filters: $656 \mathrm{~N}, 547 \mathrm{M}, 502 \mathrm{~N}, 336 \mathrm{~W}$ and $170 \mathrm{~W}$.

\section{Results}

The ISM in NGC 604 is presently at an intermediate evolutionary stage, having suffered important changes since the first massive stars formed, but not having completed the dispersal of the original cloud. The Balmer-ratio map shows that the region around the main cluster is almost devoid of dust, while some dust clouds are present at the borders of the surrounding $\mathrm{H} \alpha$ shell. This distribution is very similar to the one in the NW complex of NGC 4214 (Maíz-Apellániz et al. 1998) and indicates that the gas has been swept into a shell and that the dust in that shell has been partially destroyed by the ionizing continuum. This evolutionary state is characteristic of 3-5 Myr old starbursts. On the other hand, the $\mathrm{H} \alpha$ knot located $15^{\prime \prime}$ to the SE of the main cluster seems to be of a different character. It is very bright, located outside the main shell and seems to be 
ionized in situ by a small cluster of stars, none of them being a WR. It also has a large $W(\mathrm{H} \beta)$ and there is a dust cloud centered a short distance (1-2 pc) away. This distribution is very similar to the one in the SE complex of NGC 4214 (Maíz-Apellániz et al. 1998), implying a younger age (2-3 Myr) for that cluster. A N-S density gradient can be observed in the [S II] $\lambda 6717 /[\mathrm{S} \mathrm{II}] \lambda 6731$ map. This is consistent with the present position of the molecular cloud, which is centered $\sim 25^{\prime \prime}$ to the SE of the main cluster (Wilson \& Scoville 19 92). The young stars are presently eroding the molecular cloud and the most dense ionized gas is the one located at its border. The only other important density maximum is located at the 2-3 Myr old cluster previously mentioned, which is consistent with its younger age. The three excitation maps ( ([S II $] \lambda 6717+[\mathrm{S} \mathrm{II}] \lambda 6731) / \mathrm{H} \alpha$, $[\mathrm{N}$ II $] \lambda 6584 / \mathrm{H} \alpha$, [O III $] \lambda 5007 / \mathrm{H} \beta$ ) show that the position of the $2-3 \mathrm{Myr}$ old cluster is also the point of highest excitation. The shell around the main cluster is also clearly visible in the excitation maps due to its relative proximity to the ionizing stars. However, at the position of other stars we do not find excitation maxima, indicating that the gas around those stars has been already swept up.

Eleven WR stars are known to exist in NGC 604 (Drissen et al. 1993). We find that WR 7 and WR 11 are located very close to a dust cloud, which may explain their dimness. We have detected the WR $\lambda 4686$ bump in five of those stars (WR 1, WR 3, WR 4, WR 5 and WR 6). Also, a wide (FWHM $\simeq 2000 \mathrm{~km} \mathrm{~s}^{-1}$ ) $\mathrm{H} \alpha$ component is detected around four WR stars (WR 3, WR 4, WR 6 and WR 12). To our knowledge, of those four cases, only one (WR 6) had been previously reported (Terlevich et al. 1996). WR 6 is, by far, the case with the strongest wide $\mathrm{H} \alpha$ flux $\left(\sim 2200 \times 10^{-17} \mathrm{erg}^{-1} \mathrm{~cm}^{-2}\right)$. The wide component is also visible in $\mathrm{H} \beta$ and in $\mathrm{He} \mathrm{I} \lambda 6678$. On the other hand, the wide components in WR 3 , WR 4 and WR 12 are much weaker $\left(300-400 \times 10^{-17} \mathrm{erg} \mathrm{s}^{-1} \mathrm{~cm}^{-2}\right)$ and the wide component is invisible in $\mathrm{H} \beta$ (probably due to the lower $\mathrm{s} / \mathrm{N}$ ratio of our blue spectra). The wide component in He I $\lambda 6678$ is barely visible in WR 4 and is not present in WR 3 and 12. However, the narrow He I $\lambda 6678$ emission in WR 12 is unusually wide $\left(\mathrm{FWHM} \simeq 260 \mathrm{~km} \mathrm{~s}^{-1}\right.$ instead of $\sim 20 \mathrm{~km} \mathrm{~s}^{-1}$ ).

\section{References}

Drissen, L., Moffat, A.F.J., Shara, M.M. 1993, AJ 105, 1400

Maíz-Apellániz, J., Mas-Hesse, J.M., Muñoz-Tuñón, C., Vílchez, J.M., Castañeda, H.O. 1998, A\&A 329, 409

Terlevich, E., Díaz, Á.I., Terlevich, R., González-Delgado, R.M., Pérez, E., García Vargas, M.L. 1996, MNRAS 279, 1219

Wilson, C.D., Scoville, N. 1992, ApJ 385, 512 